\title{
LA POSICIÓN DIALECTAL DEL EUBOICO
}

In this paper the author studies the dialectal relationships of the Euboic, especially its position within the Ionic-Attic group. For that purpose she analyses the isoglosae shared by the other dialects of the Ionic-Attic group (the Minorasiatic Ionic, the Cycladic and the Attic) as well as by the Boeotian, and she concludes that, as it was suggested by A. Bartoněk in 1970, it is necessary to modify the traditional classification of the Ionic-Attic and to distinguish three subgroups: the oriental Ionic (with two varieties: the Minorasiatic one and the Cycladic one), the Attic and the Euboic. The latter is much more closely related to the Attic and also shares some features with the Boeotian.

0. El presente trabajo es un intento de definir la situación dialectal de la lengua de Eubea dentro del grupo jónico-ático.

Además de la isla de Eubea, encontramos el euboico también en Oropo, ciudad situada frente a Eretria, y en su santuario de Anfiarao, en las colonias euboicas de Italia y Sicilia, y, con escasa y dudosa documentación, en las colonias euboicas de la Calcídica.

Nuestros conocimientos sobre la lengua de Eubea se basan casi exclusivamente en los datos proporcionados por las inscripciones, pues no fue un dialecto cultivado literariamente, ni tampoco atrajo la atención de los gramáticos antiguos, de los que nos quedan muy pocos testimonios.

La desigual distribución del material epigráfico procedente de las ciudades euboicas hace que no tengamos datos suficientes para establecer las posibles diferencias locales dentro del euboico. De las ciudades euboicas, Eretria es la que más documentos epigráficos ofrece, y con un mayor número de peculiaridades lingüísticas. El material del resto de la isla es escaso, generalmente tardio, $y$, por tanto, con influencia del ático y de la koiné. Las inscripciones oropias escritas en eretrio son muy pocas, asi como las procedentes de las colonias itálicas, pertenecientes a la época arcaica. 
En cuanto a los límites cronológicos, las primeras inscripciones euboicas datan del s. vIl a. C. El euboico es utilizado hasta el s. IV a. C., e incluso el s. III a. C., aunque en esta época la mayoría de los documentos están escritos en ático y en koiné.

\section{Posición dialectal del euboico.}

1.0. En un gran número de manuales de dialectología griega (Smyth 1894; Bechtel III, p. 30; Buck 1955, p. 10; Thumb-Scherer 1959 , p. 247 ss.; asi también Schwyzer, Gr. Gr. I, p. 86 ss.), los autores establecen una subclasificación del jónico-ático, distinguiendo, por una parte, el ático, y, por otra, el jónico, al que dividen a su vez en jónico occidental o euboico, jónico de las Cícladas y jónico de Asia Menor. Por lo tanto, consideran el euboico estrechamente unido al jónico cicládico y minorasiático, como un tercer subdialecto de este grupo.

Pero ya desde hace algún tiempo (Porzig 1952; Risch 1949 y 1955; Chadwick 1956), se ha impuesto una revisión de los criterios de clasificación de los dialectos griegos, con el resultado de que, además de los métodos de la Lingüística histórica comparada, se han aplicado a la dialectología griega los métodos propios de la Geografia dialectal. Según esta orientación, hay que ver las diferencias dialectales que hay entre diferentes áreas geográficas y, mejor que hablar de dialectos bien definidos, tratar de establecer áreas dialectales o lingüisticas definidas por haces de isoglosas; es decir, para establecer el parentesco especifico entre varios dialectos a partir de un parentesco genético común hay que ver los rasgos que comparten, y sobre todo, la naturaleza y cronología de éstos.

Por lo tanto, al estudiar la posición del euboico dentro del jónicoático, es imprescindible ver las isoglosas que comparte con cada uno de los otros tres subgrupos, y los rasgos que son exclusivos suyos.

También es fundamental el hecho de que esos rasgos comunes sean arcaísmos, que carecen de valor para establecer las relaciones genéticas entre dos lenguas; opciones, que pueden ser debidas a una relación entre dos lenguas, o ser independientes en cada una de ellas; y, por último, que sean innovaciones, que son los rasgos más significativos a la hora de relacionar dos dialectos. Pero también es preciso distinguir si las innovaciones son antiguas o recientes, producidas por contactos muy posteriores a la formación de esos dialectos. Las innovaciones antiguas son, por tanto, las que indican una relación más estrecha entre dos lenguas.

Ya A. Bartoněk (1970 y 1979) hace una nueva clasificación del jóni- 
co-ático, basándose en las afinidades y diferencias de sus cuatro subgrupos, y llega a la conclusión de que el jónico de las Cícladas y el de Asia Menor están estrechamente relacionados, hasta el punto de que casi pueden ser considerados como un mismo dialecto, pues solamente presentan dos variantes (la aspiración inicial y el tratamiento de la labiovelar en el tema de interrogativo-indefinido $\left.{ }^{*}(o) k^{u} o-\right)$, y su relación con el euboico y el ático se reduce casi exclusivamente a arcaísmos.

Por otra parte, A. Bartoněk piensa también que es erróneo considerar el euboico como un subgrupo del jónico, o como un dialecto intermedio entre el ático y el jónico cicládico y el minorasiático. El análisis de sus rasgos muestra que ocupa una posición semejante a la del ático y el jónico oriental, e, incluso, está más cercano al ático, con el que comparte una serie de innovaciones, que al jónico cicládico y minorasiático, cuyos rasgos comunes son, en su mayoria, arcaísmos. Por tanto, sería más correcto hablar de jónico-euboico-ático, que de jónico-ático.

A. Bartoněk destaca también la influencia del beocio sobre el euboico. Seguramente la intensidad de esta influencia puede haber sido diferente en cada ciudad euboica, pero la escasez del material conservado nos impide saberlo con certeza.

Según A. Bartoněk, puede considerarse que el ático ocupa una posición especial, pues desarrolla una serie de innovaciones no compartidas por los otros tres grupos (solamente algunas por el euboico).

Para terminar, A. Bartoněk concluye que es errónea la clasificación tradicional del jónico-ático en dos subgrupos, por una parte el ático, y por otra, el jónico, que incluiría el euboico, el jónico cicládico y el jónico minorasiático. El término jónico-ático sólo puede ser aplicado como referencia a una comunidad lingüistica que se extiende desde el Ática hasta las costas de Asia Menor, incluyendo Eubea y las Cícladas, y en el que, alrededor del 350 a. C., podemos distinguir tres dialectos diferentes: el ático, el euboico y el jónico minorasiático (del que sería una variante el jónico cicládico).

Por nuestra parte, al estudiar los rasgos comunes del euboico con los otros tres dialectos jónicos, así como con el beocio, hemos incluido algunos rasgos no estudiados por $\mathrm{A}$. Bartoněk, $\mathrm{y}$ no hemos tenido en cuenta otros que este autor considera euboicos, erróneamente. Así:

- No hay pruebas suficientes para postular en euboico la conservación de la pronunciación posterior de $/ \mathrm{u} /$ después de la época arcaica (cf. M. del Barrio 1987, p. 221 ss.).

- En las inscripciones euboicas no aparece nunca el genitivo sin-

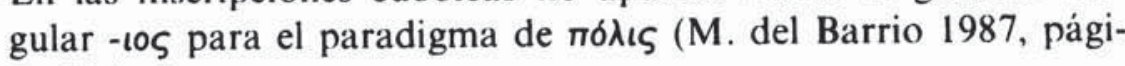
na $384 \mathrm{~s}$.). 
- Lo que A. Bartoněk considera como subjuntivos de aoristo con vocal breve, pensamos que son en realidad otra manifestación de la abreviación de los diptongos largos finales $-\eta \iota,-\omega \iota$, característica del euboico.

- La escasez de datos nos impide postular la existencia en euboico del pronombre demostrativo ékeĩvos.

\subsection{Rasgos comunes del euboico, el jónico de las Cícladas y el} jónico de Asia Menor.

1.1.1. No retroversión de $/ \bar{a} /:$ IG XII 9, 187A (Eretria, decreto de

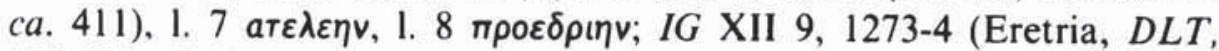

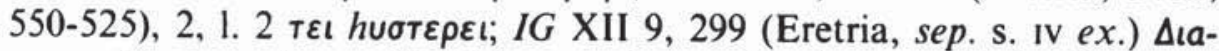

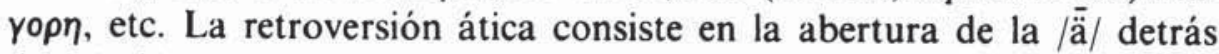
de $/ \check{e} /, / \mathrm{i} /, / \mathrm{r} /$, y su paso a $/ \bar{a} /$. Por tanto, la ausencia de retroversión supone un arcaísmo en el euboico y en el jónico de las Cícladas y de Asia Menor_con respecto al ático (independientemente de que el paso de la $/ \bar{a} /$ a /ä/ sea una innovación de todo el grupo jónico-ático respecto a los demás dialectos griegos).

1.1.2. No contracción de los grupos $\varepsilon a, \varepsilon 0, \varepsilon \circ u, \varepsilon \omega$ : $S G D I 5270(\mathrm{Cu}-$ mas, defixio, i450-425?), 1. 2 aт $\varepsilon \lambda\{\lambda\} \varepsilon a$ кai $\varepsilon \pi<\varepsilon>a$; IG XII 9, 189

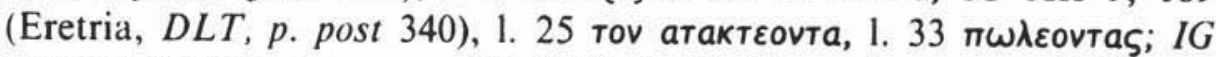

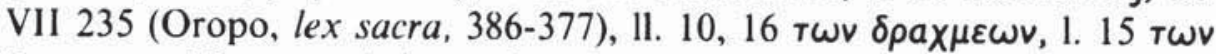
$\delta \eta \mu о \tau \varepsilon \omega v$. El mantenimiento de estos grupos es un arcaismo respecto a su contracción.

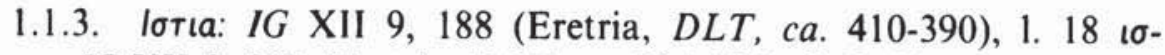

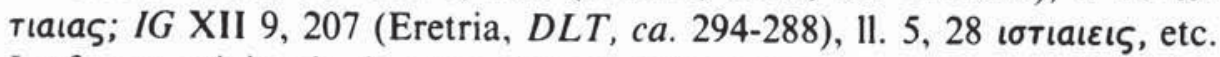
La forma originaria de esta raiz $\varepsilon \sigma \tau$ - solamente se conserva en ático; todos los demás dialectos griegos han innovado, incluido el euboico (C. D. Buck 1955, parágr. 11, p. 23).

1.1.4. Desinencia $-\varepsilon \omega /-\omega$ en el genitivo singular de los masculinos en -ā. El griego innovó en la desinencia del genitivo singular de los masculinos en $-\bar{a}$, y adoptó la terminación ${ }^{*}-a \bar{o}$, en lugar de ${ }^{*}$-äs, por analo-

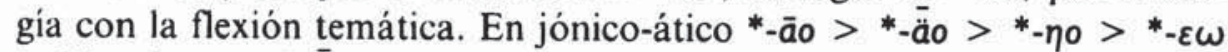
(o bien *-āo $>*^{*}-\bar{a} o>*^{*}$-āo $>-\varepsilon \omega$, según la cronología de la metátesis de cantidad; vid. E. Crespo 1977). Posteriormente el ático innovó, introduciendo la desinencia -ou de genitivo de la flexión temática después de la contracción de *-oo (vid. E. Schwyzer, Gr. Gr. I, p. 561; Chantraine 1974 , p. 36; Buck 1955, p. 87, parágr. 105,2; Szemerényi, Glotta 35 , 
p. 196 ss.). Por lo tanto la desinencia - $\varepsilon \omega$ del euboico y del jónico cicládico y minorasiático es un arcaísmo en estos dialectos respecto al ático. Algunos ejemplos aparecen en: $B E 1964, n .^{\circ} 46$ (Eretria, ded. s. v ex.)

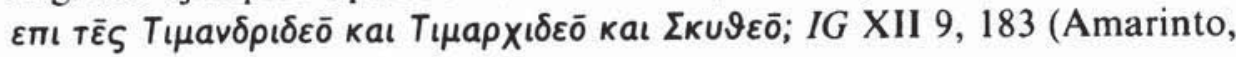

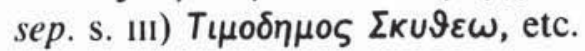

1.1.5. Desinencia -Eos en el genitivo singular de los temas en -s-:

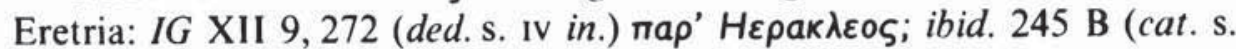

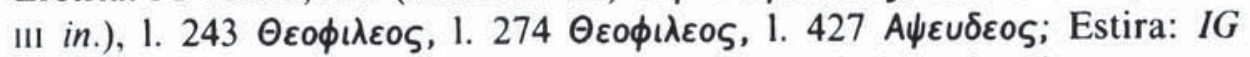

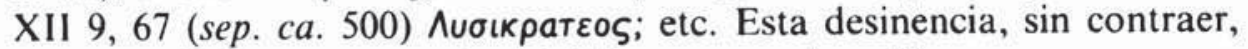
es un arcaísmo respecto a la del ático, con contracción.

1.1.6. Genitivo singular noגعos. En las inscripciones euboicas encontramos noגعos en Oropo, en $I G$ VII 235, lex sacra de $c a$. 386-377, con abreviación de la $\eta$ sin metátesis de cantidad, quizá por la influencia de la desinencia de genitivo sg. - $\varepsilon \circ \varsigma$ de los temas en $-s$ - (así, ThumbScherer 1959 , p. 272). Respecto a la existencia de un tema en laringal en noגıs, vid. M. Ruipérez 1986.

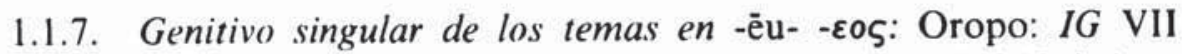

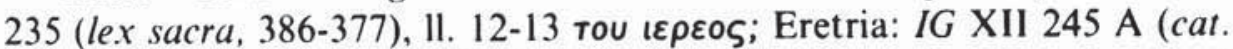

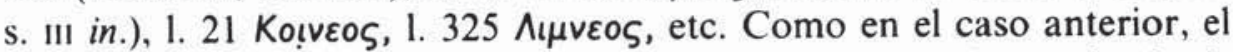
origen de estas formas está en la abreviación de la $\eta$ sin metátesis de cantidad por influencia del genitivo de los temas en $-s$ - (Thumb-Scherer 1959, p. 273).

1.1.8. Forma del comparativo $\mu \varepsilon$ לov-. Esta forma aparece una vez en Oropo, en $I G$ VII 235 , lex sacra de 386-377, $\mu \varepsilon \zeta$ ova, y es un arcaísmo respecto a la forma del ático $\mu \varepsilon ı \zeta o v-$.

1.1.9. Forma عovт- del participio presente de elpí: Oropo: IG VII 235 (lex sacra, 386-377), II. 12, 23-24 пареovтos; $A E$ 1917, n. 94 (s.f.), 1. 10 Eоvтоs.

Por lo tanto, vemos que la mayoría de los rasgos que comparten el euboico y el jónico cicládico y minorasiático son arcaísmos. Las tres innovaciones que tienen en común son debidas a la analogía (la 1.1.3 se da en todos los dialectos griegos menos en ático), y no indican una relación especial entre los tres subgrupos del jónico-ático.

1.2. Rasgos comunes del euboico, el ático y el jónico de las Cícladas.

1.2.1. Conservación de la aspiración inicial: Disto: $I G$ XII Suppl. 78 (mojón, ss. v-Iv) hopos; Eretria: $I G$ XII 9, 1273-4 (DLT, i550-525?), 2, 


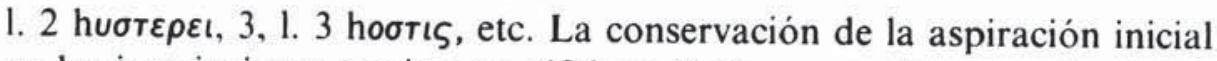
en las inscripciones escritas en alfabeto jónico se puede ver en los casos de elisión como $\varepsilon \phi^{\prime}$ เтпоч (IG XII 9, 196, Eretria, $D L T$, s. IV ex., 1. 8),

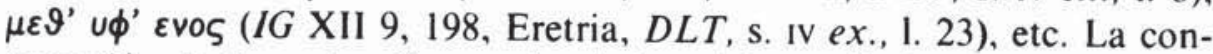
servación de la aspiración en el euboico, ático y jónico cicládico es un arcaísmo, frente a la psilosis del jónico minorasiático.

1.2.2. Forma en (0)mo- en el interrogativo-indefinido. Frente a las formas en (o)ko- que aparecen en jónico minorasiático (Bechtel III, pp. 87-89; Thumb-Scherer 1959, p. 62), las inscripciones euboicas presentan siempre (o)חo-, como el ático y el jónico cicládico: Eretria, $I G$ XII 9. 188 (DLT, ca. 410-390), 1. 5 опораı, 1. 10 опотеро , etc. La explicación más probable (Lejeune 1972, p. 45) es la existencia en protogriego de las dos formas negativas *ou- $k^{u-}-\bar{s}$, que después dará ouk $\omega \varsigma$, por disimilación de la labiovelar ante la $u$ precedente, $\mathrm{y}^{*} m \bar{e}-k^{*-} \bar{o} s>\mu \eta \pi \omega \varsigma$. Después los dialectos regularizaron una de las dos formas, por lo que nos encontramos ante un caso de opción.

1.2.3. Desinencia del dativo plural temático -oıs. La única forma atestiguada en las inscripciones euboicas en -oเs, Oropo, IG VII 235

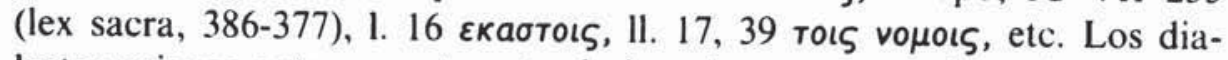
lectos griegos optaron entre esta desinencia, que parte de un instrumental indoeuropeo ${ }^{*}-\bar{o} i s$, y un antiguo locativo, *-oisi (Chantraine 1974, parágr. 20).

Por tanto, tampoco los rasgos compartidos por el euboico, el ático y el jónico cicládico suponen una relación especial entre estos tres dialectos frente al jónico minorasiático, después de la migración jónica, pues o bien son arcaísmos, $u$ opciones que pueden darse de manera independiente en varios dialectos.

\subsection{Rasgos comunes del euboico y del ático.}

1.3.1. Abreviación del diptongo $-\eta \mathbf{t}$ en posición final: la abreviación de $-\omega$ ı en ático se da sólo en casos aislados (Threatte 1980, parágr. 17.03); la de $-\eta \iota$ comienza a partir de fines del s. $v$, pero es frecuente sobre todo desde 375-350 a. C. (Threatte 1980, parágr. 23). En euboico, esta innovación data también de fines del s. v: Eretria, $I G$ XII 9, $187 \mathrm{~A}$

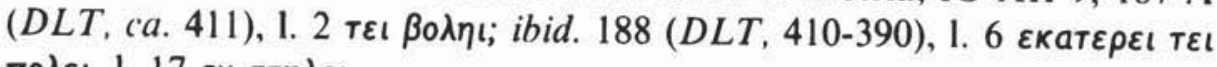
по入 $\varepsilon \iota, 1.17 \varepsilon v \sigma \tau \eta \lambda \varepsilon \iota$.

1.3.2. Nombres en - $\mathrm{k} \lambda \varepsilon \eta \mathrm{S}$ sin contraer: estos nombres contrajeron tempranamente en ático y euboico. Posteriormente, por analogía con el 
acusativo y el genitivo se volvió a crear un nominativo en $-\kappa \lambda \varepsilon \eta \varsigma$ (cf. Thumb-Scherer 1959, parágr. 321, 6b, p. 292; Buck 1955, parágr. 108, Ia). Que las formas en $-k \lambda \varepsilon \eta \varsigma$ son más recientes que las contractas lo podemos ver en las inscripciones euboicas, donde los ejemplos más antiguos presentan contracción (SGDI 5294, 5298A, 5300, vasos calcídicos,

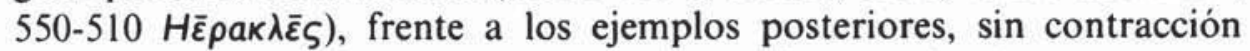

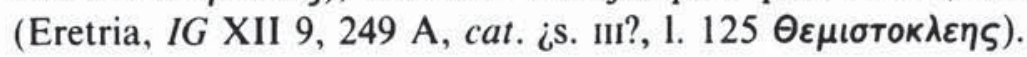

1.3.3. Ausencia del tercer alargamiento compensatorio: Eretria, IG XII 9, 187 (DLT, s. IV in.), 1. $13 \pi \rho \circ \xi \varepsilon v o v, 1.18 \pi \rho \circ \xi \varepsilon v o ı s, ~ e t c . ~ L a ~ a p a r i-$ ción o no del tercer alargamiento compensatorio está ligada a un problema de silabación; según se pronuncie ${ }^{*} \kappa a \lambda-F \circ \zeta$, o ${ }^{*} \kappa a-\lambda F \circ S$, el grupo ${ }^{*}-\lambda-F$ hará posición y alargará la sílaba precedente, o no lo hará; en el primer caso, al desaparecer la $F$ la cantidad larga de la sílaba anterior será mantenida mediante el alargamiento compensatorio de la vocal. Por tanto, este rasgo está estrechamente emparentado con la correptio attica. En el caso del euboico, al no disponer de textos literarios en metro (pues las inscripciones métricas euboicas utilizan una lengua épica), no podemos saber si se daba también la correptio; solamente, mediante el testimonio indirecto de la ausencia del tercer alargamiento, hemos de suponer que tampoco en euboico los grupos ${ }^{*}-\rho_{F^{-}},-\lambda_{F}-$, etc., hacian posición.

1.3.4. Forma เepos. Es la única forma atestiguada en las inscripciones euboicas: Eretria, $I G$ XII Suppl. 549 B ( $D L T$, ca. 500-475), 1. 6 hıє-

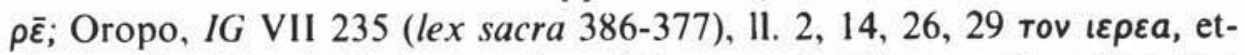
cétera. El origen incierto de esta palabra nos impide considerarla como arcaísmo, opción o innovación.

1.3.5. $\mathrm{rs}>\mathrm{rr}$ : los primeros ejemplos en las inscripciones euboicas datan de la segunda mitad del s. vII, en las colonias de Italia y Sicilia: Leontinos, SEG IV 64 (fragmento de ley sobre homicidios, ca. 525) B,

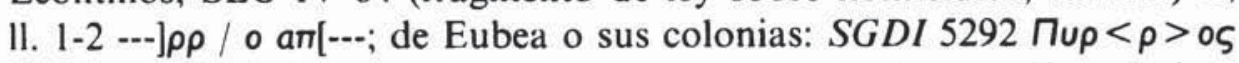
(segunda mitad s. viI). Se trata, por tanto, de una innovación relativamente antigua en euboico. En ático los primeros testimonios son de finales del s. vi (vid. Threatte 1980, parágr. 43.03).

1.3.6. -tt-, resultado de *-t(h)i-, *-k(h)i-, *-tu-, y labiovelar sorda más yod. En las inscripciones áticas está atestiguado desde el s. vi (vid. Threatte 1980, parágr. 43.04). En euboico no aparece hasta $c a .475$, en Estira, IG XII 9. 56 (tablillas de plomo) n. ${ }^{\circ} 183$ Kıтtı̌s, , pero seguramente el fenómeno es anterior. Por otra parte, $-t t$ - aparece en inscripciones con numerosos rasgos dialectales, por lo que podemos conside- 
rarlo como una característica propia del euboico. Además del ático y del euboico, - $t t$ - se encuentra también en beocio y cretense, más algunos ejemplos tesalios y etolios (vid. Buck 1955, parágr. 81; Blümel 1982, p. 118 ss.; M. Dosuna 1985, pp. 126-128).

Por la diferencia de resultados $s s / t t$ en los dialectos griegos, hemos de admitir en protogriego un estadio intermedio ${ }^{*}-t s^{-}$, que todavía existía cuando se fragmentaron los grupos eólico y jónico-ático, lo que explicaría el resultado diferente del beocio, el euboico y el ático, respecto al lesbio y al jónico cicládico y minorasiático, con asimilación progresiva en unos, y regresiva en los otros. Por otra parte, en jónico-ático (y en arcado-chipriota) anteriormente se habia producido una primera oleada de palatalización, que afectó a los grupos ${ }^{*}-t(h) y$ - en los que la oclusiva y la yod no estaban separadas por un «limite de morfema». Estos grupos dieron ${ }^{*}-t s-$, que se confundió con ${ }^{*}-t s$ - originario, y ambos evolucionaron a ${ }^{*}-s s-$, por lo que no se confundieron con ${ }^{*}-t s$ - resultado de la segunda palatalización que afectó a los restantes grupos *-t(h)y- (vid. Brixhe 1982, p. 210 ss.).

1.3.7. Desinencia -aıs en el dativo plural de los temas en -ã. Los ejemplos euboicos no son muy numerosos, pero ya aparecen en Regio en la primera mitad del s. v, así como en las inscripciones dialectales de

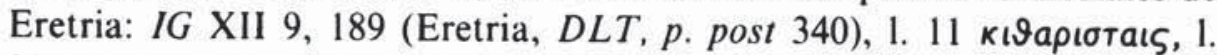

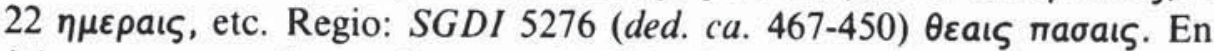
ático aparece sobre todo a partir del 420 a. C. (vid. Thumb-Scherer 1959, pp. 239-294, parágr. 322.2). La desinencia -aıs es analógica de la del dativo plural temático -oıs (vid. Chantraine 1974, parágr. 36, p. 33).

1.3.8. Paso de los antropónimos de la flexión -ı5, เo5, a la flexión

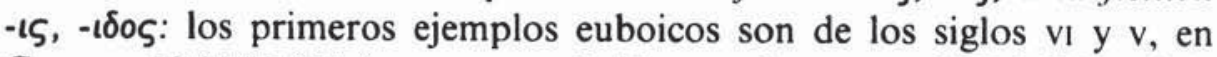
Cumas, IG XIV 867 (sep. s. vi ex.) $\Delta \bar{\varepsilon} \mu o x a \rho ı \delta$ s, y en SGDI 5270 (defixio, ¿ca. 450-425?), 11. 1, 3 0röpıos. Este cambio de flexión en los antropónimos masculinos es también frecuente en ático (vid. Buck 1955, parágr. 109,5$)$.

1.3.9. Genitivo plural femenino toutwv: Eretria, IG XII 9, 189 ( $D L T$, p. post 340 ), 11. 7-8 toutwv. Este rasgo se trata de una innovación analógica del tema demostrativo del masculino, en un intento de regularizar el paradigma. En ático esta regularización sólo alcanza al genitivo plural femenino rout $\omega v$, mientras que en euboico se extiende también a otros casos, y está atestiguada ya en el s. vi en las colonias itálicas (Cumas, IG XIV 817 sep., ¿ca. 525-500?, тоuтعı, dativo sg. femenino). Este rasgo parece ser una isoglosa de Grecia central, pues se da 
también en beocio, délfico y locrio epizefirio (vid. M. Dosuna 1985, p. 195; Chantraine 1974, parágr. 135).

1.3.10. Numeral $\chi \iota \lambda \iota$ เo. Es la única forma atestiguada en las inscripciones euboicas (Eretria, IG XII 9, 189, DLT, p. post 340, 1. 5 xt$\lambda \iota \omega v$, etc.). Se trata de una asimilación de la /ẹ/ a la iota posterior (vid.

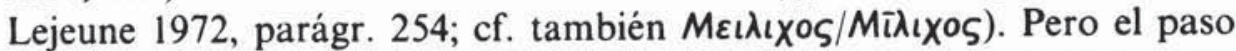
de $/ \bar{e} /$ a $/ \mathbf{i} /$ se produce también en otras palabras sin la secuencia $-\bar{\varepsilon} \lambda_{t}$ (cf. вiцатіоv/ïarıv). Sobre el origen del fenómeno, vid. Wackernagel, $K l$. Schr., II, p. 1025, que lo atribuye a la influencia de una capa social baja.

1.3.11. Sufijo $-\vartheta \varepsilon(v)$ para formar adverbios de lugar a partir de topónimos. En euboico solamente está atestiguado en Eretria, a partir del

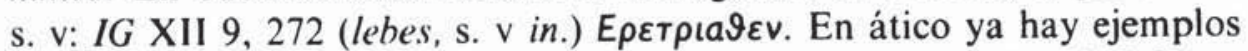
también en el s. v, pero es más frecuente en el s. Iv (vid. Thumb-Scherer 1959, parágr. 323, 3a, p. 296).

Por lo tanto, vemos que la mayoría de los rasgos comunes del euboico y del ático son innovaciones. Algunas de éstas son antiguas, anteriores al s. v $(1.3 .5,1.3 .6,1.3 .8,1.3 .9)$; otras son datables en el s. v, o posteriores $(1.3 .1,1.3 .2,1.3 .7,1.3 .10,1.3 .11)$.

\subsection{Rasgos comunes del euboico y del beocio.}

1.4.1. Abreviación del diptongo final - $\omega t$ : Eretria, IG XII 9, 189 (DLT, p. post 340), Il. 16, 17, 19 трเтоเ, 11. 31, 41, то เєроเ, etc. La abreviación de los diptongos largos finales es una isoglosa que se extiende por una gran zona de Grecia central: Ática, Beocia, Tesalia occidental, y dialectos del Noroeste, llegando hasta el Epiro por el norte, y hasta Élide y Arcadia en el Peloponeso (cf. Thumb-Scherer 1959, parágr. 237, 2; 246, 2b; Lillo 1981; Blümel 1982, parágr. 62, 253; M. Dosuna 1985, p. 450 ss.).

1.4.2. $\varepsilon a>$ เa. Es un fenómeno propio del sur de Beocia, donde ya aparece en las inscripciones arcaicas y en Corina, y del dórico (vid. Buck 1955, parágr. 9; Blümel 1982, parágr. 42 ss., y 1985, parágr. 2, 2). En euboico sólo está atestiguado en Eretria y Leontinos, en dos inscripciones que datan de la misma época, la segunda mitad del s. vI: $I G$ XII 9, 1273-4, y SEG 4, 64 เav.

1.4.3. Iвро५. No está claro si en beocio la forma originaria era

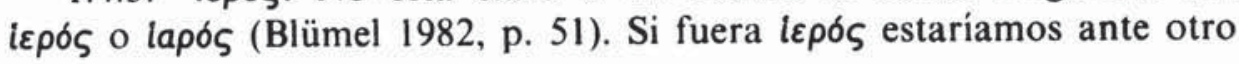


rasgo compartido por el euboico, el ático y el beocio. En tesalio también encontramos la forma iepós (vid. García Ramón 1975, pp. 54-55).

1.4.4. -tt- resultado de *-t(h)i-, *-k(h)i-, *-tul-, y labiovelar sorda más yod. Este rasgo aparece también en ático, cretense, y en algunos ejemplos tesalios y etolios (vid. 1.3.6).

1.4.5. Frecuencia de los sufijos - $\omega v \delta \eta \bar{S}$, - $\ \bigcirc \bigcirc$ en los antropónimos:

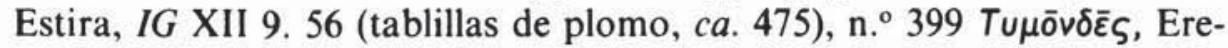
tria, IG XII 9. 245 A (cat. s. III in.), 1. 7 Фıגovixos, 11. 208, 209 Фpuvt-

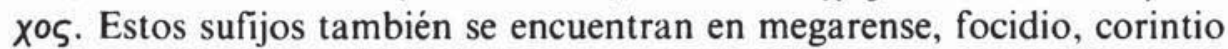
y tesalio; - $\$ ×0S es frecuente en ático. Por lo tanto, parece que se trata de una isoglosa de esta zona de Grecia central (vid. Buck 1955, parágr. 164,8 y 9 ).

1.4.6. Extensión analógica del tema demostrativo del masculino, тоUт- en euboico, -out- en beocio. El fenómeno se da también parcialmente en délfico, locrio epizefirio y ático (vid. Chantraine 1974, parágr. 135; M. Dosuna 1985, p. 195). Es posible que la innovación partiera del beocio, donde ya se encuentra en las inscripciones arcaicas y en Corina (cf. Diehl, 5, 80), y donde la regularización fue mayor, ya que el tema del nominativo sg. masculino se generalizó en el resto del paradigma. Sobre la posibilidad de que las formas beocias sean arcaísmos, y los demás dialectos griegos hayan innovado cambiando la primera sílaba por analogía con las formas correspondientes del artículo, vid. C. Watkins, Celtica 6, 1963, p. 22 ss., al que sigue Blümel 1985, p. 391.

1.4.7. Fórmula $\varepsilon \Pi \iota+$ dativo en las estelas sepulcrales: Eretria, $I G$

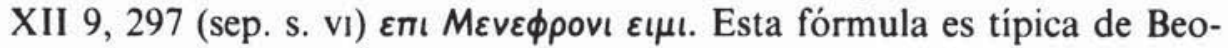
cia, pero aparece también en las regiones limitrofes de la Fócide y Lócride (Buck 1955, parágr. 136, 6; M. Dosuna 1985, pp. 29 y 245; Thumb-Scherer 1959, parágr. 238, 6b).

Así pues, vemos que la mayoría de los rasgos comunes del euboico y del beocio no se limitan a estos dos dialectos, sino que también aparecen en otros dialectos vecinos de Grecia central.

El rasgo más extendido geográficamente es la abreviación de los diptongos largos finales $-\bar{a} \iota>-\check{a}_{\imath},-\eta \iota>-\varepsilon \iota,-\omega \iota>-o \iota$, con variaciones en los diferentes dialectos: en euboico $-\eta \iota>-\varepsilon \iota,-\omega \iota>-o l$; en ático solamente $-\eta \iota>-\varepsilon \iota$, y aisladamente $-\omega \iota>-o l$; en beocio, $-\bar{a} \iota>-a ̆ ı, ~-\omega \iota$ $>-o l$; en los demás dialectos sólo es seguro el paso $-\omega \iota>-o \iota$, ya que -äı > -ăı no puede ser registrado por la ambigüedad del signo $A$.

Otros rasgos ( $\varepsilon \pi \iota+$ dativo, los sufijos $-\omega v \delta a \bar{s},-\iota \times \circ \varsigma)$ son propiamente beocios, pero se han extendido a algunas de las regiones limitro- 
fes, o bien se realizan en beocio de una manera más amplia que en los demás dialectos vecinos, como, por ejemplo, la extensión analógica del tema de demostrativo del masculino.

En cuanto a $-t t$-, este rasgo se da en beocio, ático y euboico, en Grecia central; los ejemplos tesalios y etolios no son seguros (vid. 1.3.6). No podemos decir con certeza el lugar de origen del fenómeno, pero es probable que se iniciara en el beocio, donde - $t$ - tiene su correspondencia en la serie sonora, $-d d-$, procedente de la evolución ${ }^{*}-d y$-, ${ }^{*}$-gy-, mientras que el ático y el euboico presentan el mismo resultado para estos grupos que el resto del jónico-ático, /zz/.

De estos rasgos comunes al euboico y al beocio, unos están atestiguados en las inscripciones euboicas ya en el s. vı (el paso $\varepsilon a>\iota a$, la extensión del tema de demostrativo masculino, y la fórmula $\varepsilon \pi \iota+$ dativo en las estelas sepulcrales); otros, en el primer cuarto del s. v, aunque quizá son más antiguos $\left(-t t-\right.$, y los sufijos $-\omega v \delta \eta \varsigma$ e $-t \chi \chi^{\circ}$ en antropónimos). El más reciente es la abreviación del diptongo $-\omega \iota>-o \iota$, que en Eubea data de finales del s. $v$ o comienzos del s. Iv, y en Beocia del s. IV.

Según esto, podemos pensar que el euboico y el beocio estuvieron relacionados desde el s. vı, o quizá antes, hasta finales del s. v o comienzos del s. IV.

\section{Conclusiones.}

Del análisis de los rasgos mencionados podemos concluir lo siguiente:

- No hay ningún rasgo común del euboico con el jónico cicládico que sea exclusivo de estos dos dialectos, ni tampoco exclusivo del euboico con el jónico minorasiático. Esta característica ya había sido señalada por A. Bartoněk en 1970 y 1979.

- La mayoría de los rasgos comunes del euboico, el jónico de las Cícladas y el jónico de Asia Menor, son arcaísmos. Las pocas innovaciones que comparten son debidas a la analogía (algunas de las cuales aparecen también en otros dialectos), y, por tanto, no implican que haya habido una relación entre el euboico y los otros dos dialectos jónicos, independientemente del ático, después de la migración jónica.

- Los rasgos comunes al euboico, ático y jónico cicládico son arcaísmos o elecciones, $y$, por tanto, tampoco suponen una relación entre estos tres dialectos después de la fragmentación del jónico-ático.

-De los rasgos comunes al euboico y al ático, y que no son compartidos por los otros dos dialectos jónicos, la mayoría son innovacio- 
nes. Como ya hemos visto, algunas de ellas son anteriores al s. v, pues ya hay ejemplos en euboico en los s. VII y vi, y su carácter antiguo está apoyado, además, por el hecho de que aparecen en las colonias euboicas de Italia y Sicilia; otras no están atestiguadas en las inscripciones euboicas hasta el s. v, pero seguramente son anteriores, pues en ático ya aparecen en el s. vi; $y$ otras datan de finales del s. v, o del s. Iv.

Estas innovaciones comunes implican una conexión entre el euboico y el ático posterior a la migración jónica, pues no se producen en las Cícladas ni en Asia Menor, pero anterior a los ss. v y iv, cuando Atenas interviene en la vida política de Eubea, y el ático comienza a desplazar al euboico, como pasó también con otros dialectos.

- Por otra parte, dentro del jónico-ático el euboico ocupa una posición especial, por su relación con el beocio. Algunos de los rasgos que tienen en común aparecen también en otros dialectos vecinos, incluido el ático, por lo que se trata de isoglosas que afectan a esta zona de Grecia central. La cronología de estas características comunes va desde el s. VI, fecha en que aparecen los primeros testimonios, aunque el fenómeno puede ser anterior, hasta comienzos del s. IV.

- Por tanto, el resultado de nuestro análisis de los rasgos euboicos coincide con las observaciones realizadas por A. Bartoněk: no tenemos pruebas de una evolución conjunta del euboico y el jónico cicládico y minorasiático; sí las hay, en cambio, de una relación entre el euboico y el ático, pues comparten una serie de innovaciones posteriores a la migración jónica, y que no se dan en los otros dos grupos del jónico-ático, así como de una relación entre el euboico y el beocio.

Asi pues, también nosotros pensamos que hay que variar la clasificación tradicional del jónico-ático: el euboico no es ningún subgrupo del jónico, ni es un dialecto intermedio entre éste y el ático, sino que ocupa una posición semejante a la de estos dos dialectos, más próxima al ático, con el que comparte una serie de innovaciones posteriores a la migración jónica.

Por otra parte, el eretrio (quizá todo el euboico) presenta unos desarrollos propios específicos, como son el rotacismo intervocálico, los adverbios de lugar опоь, $\eta \chi \circ \iota$, así como una mayor frecuencia del paso de los verbos en $-\mu$ t a la flexión de los verbos contractos en las formas no personales, mientras que en otros dialectos que presentan también este rasgo, se da sobre todo en las formas personales. Si estas características se limitan al eretrio, o si eran propias también de la lengua de las demás ciudades euboicas, no podemos saberlo, por la escasez del material epigráfico conservado. 
3. Interpretación de las relaciones dialectales del euboico.

Como ya hemos visto en el apartado anterior, las afinidades del euboico con el ático y el beocio nos llevan a la conclusión de que estos tres dialectos estuvieron en contacto después de la migración jónica. Pero ¿en qué momento de su historia hay que situar esta conexión? Vamos a intentar hacer una hipotética reconstrucción de lo que pudo haber sido la evolución del euboico después de la separación del jónicoático.

Generalmente se admite que a finales del segundo milenio antes de Cristo, o comienzos del primero, tuvo lugar la migración jónica, por la presión de otros pueblos sobre el Ática, y que supone el inicio de la fragmentación del jónico-ático (J. Mangas 1981, pp. 35-36).

Antes de la migración, los jónico-áticos se concentraban principalmente en el Ática, seguramente también en el sur de Beocia, y ocupaban también la parte central de la costa occidental de Eubea. Cuando se produjo la migración, se extendieron por toda la isla, y partieron hacia las Cícladas y Asia Menor (Thumb-Kieckers 1932, p. 68; Tovar 1944, p. 244 ss.; Porzig 1952, p. 164; Thumb-Scherer 1959, p. 15; Buck 1968 , pp. $268-271$ y 276 ss.). En el momento de producirse la migración, el jónico-ático conservaba todavía la pronunciación de la /u/ como vocal posterior, $\mathrm{y}^{*}$ - $t s$ - resultado de la evolución de los grupos ${ }^{*}-t(h) y_{-},{ }^{*}-k(h) y-,{ }^{*}-t w^{-}$, y labiovelar más yod. No se había producido todavía la contracción de los grupos formados por /e/ más una vocal diferente, ni la asimilación de $-r s^{-}$. Ya había tenido lugar el paso de $/ \bar{a} /$ a /ä/, pero no el de $/ \bar{a} /$ a $/ \bar{e} /$, ni la retroversión ática. La forma de comparativo era $\mu \varepsilon \zeta$ - La desinencia del genitivo singular de los masculinos en $-\bar{a}$ era $-\varepsilon \omega$ (o mejor $-\bar{a} o)$, y la de los temas en $-s-,-\varepsilon \circ \varsigma$. Para el dativo plural temático se usaban las dos desinencias -oıs y -oเoı. Todavía no se había producido el paso de la flexión $-15,-105$ a la de $-15,-1 \delta 05$.

A partir de entonces el jónico de las Cícladas y el de Asia Menor evolucionaron separadamente del ático y del euboico, pues casi todos los rasgos que tienen en común son arcaísmos, procedentes del estadio de comunidad jónico-ática. Las pocas innovaciones posteriores que comparten son desarrollados paralelos, independientes, y debidos a la analogía, como el empleo de formas temáticas para los verbos atemáticos, más algún caso de opción, que no implican necesariamente una relación entre dos dialectos.

Las innovaciones antiguas, atestiguadas desde los ss. vII y vI, comunes al euboico y al ático, hacen suponer que hubo un contacto entre es- 
tos dos dialectos anterior al s. vi. Pero ¿en cuál de las dos lenguas se originaron primero, desde la cual se extendieron a la otra?

En primer lugar, una influencia del ático sobre el euboico sólo es pensable a partir de finales del s. VI, cuando comienza la ascensión politica de Atenas, mientras que Eubea está en decadencia. Es poco probable que hubiera una influencia del ático sobre el euboico en los ss. vIII y vil, y que explicaría las innovaciones comunes de esta época, sino que más bien sería al contrario.

En efecto, en los ss. VIII y vil las ciudades euboicas están en su apogeo político (es cuando tiene lugar la colonización euboica de Italia y Sicilia, y el dominio de Eubea sobre las Cícladas, e incluso sobre parte del continente, como, por ejemplo, Oropo, Aulis y Kanethos), y tienen una situación más importante que la de Atenas (que no participa en la colonización) (vid. K. Schefold-P. Auberson 1972, p. 19 ss.). Por lo tanto, es posible pensar que algunas de las innovaciones comunes tuvieron su origen en Eubea, desde donde pasaron al ático.

Después de la guerra lelantina comienza la decadencia de las ciudades euboicas. A finales del s. vII o comienzos del s. vI, Eretria pierde Oropo ante los tebanos, y seguramente Eubea entra bajo la influencia de Beocia. Probablemente de esta época son los rasgos beocios que aparecen en euboico $(\varepsilon a>\imath a ;-\omega v \delta \eta \varsigma,-\iota \chi \circ \varsigma ; \varepsilon \pi \iota+$ dativo en las estelas sepulcrales).

Desde comienzos del s. v la importancia de Atenas crece, así como su influencia política sobre Eubea, que se ve alcanzada por el imperialismo ateniense. De esta época son los establecimientos de clerucos atenienses en diversas ciudades de Eubea, y la pertenencia de ésta a la Liga de Delos.

Es lógico que la influencia de Atenas también se haya notado en el terreno lingüístico, y así, se introducen algunos rasgos áticos en el dialecto de Eubea (¿sufijo $-\vartheta \varepsilon(v)$, antropónimos en $-\kappa \lambda \varepsilon \eta \zeta$ ?), hasta que, finalmente, el euboico es desplazado por el ático, sobre todo a partir del s. IV. Entonces, los eubeos utilizarán su dialecto principalmente en los períodos de ruptura con Atenas y de independencia política, como un instrumento de reacción nacional.

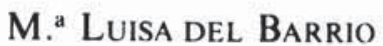

\section{REFERENCIAS BIBLIOGRÁFICAS}

Barrio, M. L. del 1987: El dialecto de Eubea. Madrid (ed. Universidad Complutense). 
Bartoněk, A. 1970: "Attic-Ionic dialects reclassified», Sbornik 19 (E 15).

Bartoněk, A. 1979: "Greek dialects between 1000 and 300 B.C.", SMEA 20: pp. 113-30.

Bechtel, F. 1924: Die griechischen Dialekte, III. Der ionische Dialekt. Berlín.

Blümel, W. 1982: Die aiolischen Dialekte. Phonologie und Morphologie der inschriftlichen Texte aus generativer Sicht. Göttingen.

Blümel, W. 1985: "Charakterisierung des boiotischen Dialekts», en Colloques internationaux du CNRS "La Béotie antique», París.

Brixhe, Cl. 1982: «Palatalisations en grec et en phrygien. Problèmes phonétiques et graphiques", BSL: pp. 209-249.

Buck, C. D. 1955: The Greek dialects: grammar, selected inscriptions, glossary. Chicago.

Buck, R. J. 1968: "The Aeolic dialect in Boeotia», Classical Philology 43, 4: pp. 268-280.

Chadwick, J. 1956: "The Greek dialects and Greek pre-history", $G \& R$ 3: pp. 38-50.

Chantraine, P. 1974: Morfologia histórica del griego. Barcelona.

Crespo, E. 1977: "La cronologia relativa de la metátesis de cantidad en jónicoático", $C F C$ 12: pp. 188-219.

García Ramón, J. L. 1975: Les origines postmycéniennes du groupe dialectal éolien. Salamanca.

Inscriptiones Graecae, VII: Inscriptiones Megaridis et Boeotiae (Inscriptiones Oropiae, pars altera), ed. G. Dittenberger 1982.

Inscriptiones Graecae, XII: Inscriptiones insularum maris Aegei praeter Delum, fasc. 9: Inscriptiones Euboeae insulae, ed. E. Ziebarth 1915.

Supplementum ad Inscriptiones Graecae, XII: fasc. 9: 174-207, ed. E. Ziebarth 1939.

Inscriptiones Graecae, XIV: Inscriptiones Siciliae et Italiae, additis Graecis Galliae, Hispaniae, Britanniae, Germaniae inscriptionibus, ed. G. Kaibel 1890.

Lejeune, M. 1972: Phonétique historique du mycénien et du grec ancien. Paris.

Lillo, A. 1981: "En torno a los dativos de la declinación temática en -o y en - $\omega$ t del arcadio", Studia Philologica Salmanticensia. 5: pp. 221-232.

Mangas, J. 1981: Textos para la historia antigua de Grecia. Madrid.

Méndez Dosuna, J. 1985: Los dialectos dorios del Noroeste. Gramática y estudio dialectal. Salamanca.

Porzig. W. 1952: «Sprachgeographische Untersuchungen zu den altgriechischen Dialekten», IF 61: pp. 147-69.

Risch, E. 1949: "Altgriechische Dialektgeographie?", MH 5: pp. 19-28.

Risch, E. 1955: "Die Gliederung der griechischen Dialekte in neuer Sicht", $M H$ 12: pp. 61-76.

Ruipérez Sánchez, M. 1986: «Observations phonétiques et morphologiques autour de пróגı૬" (en prensa). The Greek Language (Coloquio de Larnaka, septiembre).

Schefold, K., y P. Auberson 1972: Führer durch Eretria. Berna.

Schwyzer, E. 1939: Griechische Grammatik, I. Lautlehre, Worthildung, Flexion. Munich.

SGDI: H. Collitz, F. Bechtel, Sammlung der griechischen Dialekt-Inschriften. Göttingen 1894-1915.

SEG: Supplementum Epigraphicum Grac'um. Leiden 1923-1981.

Smyth, H. W. 1984: The sounds and inflections of Greek Dialects, I. Oxford.

Thumb A., y E. Kieckers 1932: Handbuch der griechischen Dialekte, I. Heidelberg.

Thumb A., y A. Scherer 1959: Handhuch der griechischen Dialekte, II. Heidelberg. 
Tovar, A. 1944: «Primitiva extensión geográfica del jonio», EMERITA 12: p. 245 ss.

Threatte L. 1980: The grammar of Attic inscriptions, I. Phonology. Berlin-Nueva York. 